Informe especial

\title{
El cólera epidémico en América Latina de 1991 a 1993: implicaciones de las definiciones de casos usadas en la vigilancia sanitaria ${ }^{1}$
}

\author{
Denise Koo, ${ }^{2}$ Héctor Traverso, ${ }^{3}$ Marlo Libel, ${ }^{4}$ \\ Christopher Drasbek, ${ }^{3}$ Robert Tauxe ${ }^{2}$ y David Brandling-Bennett ${ }^{5}$
}

RESUMEN En este informe se presentan las diversas definiciones de casos de cólera usadas en los países de América Latina que se han visto afectados por la epidemia; se da el número de casos de cólera $y$ de las defunciones por la enfermedad (según datos notificados a la OPS por los países latinoamericanos en 1993), y se describen algunas tendencias regionales de la incidencia de cólera. La información relacionada con la forma en que se definieron los casos de la enfermedad se obtuvo por medio de un cuestionario administrado por la OPS en octubre de 1993.

En total, 948429 casos de cólera fueron notificados a la OPS entre enero de 1991 y diciembre de 1993 por los países latinoamericanos afectados por la epidemia y las incidencias anuales más altas se registraron en el Perú (1991 y 1992) y Guatemala (1993). La tasa de letalidad para todo el trienio, y también para 1993, fue de 0,8\%. La incidencia de cólera mostró una tendencia descendente general en la mayor parte de los países sudamericanos pero aumentó en casi todos los países de Centroamérica.

Se observó gran variabilidad en las definiciones aplicadas para notificar casos de cólera, casos de cólera hospitalizados y defunciones atribuibles al cólera. Esta variabilidad dificulta cualquier comparación global entre países (y hasta estimar la carga de morbilidad y evaluar la calidad de la atención sobre la base de las tasas de letalidad), y aun las tendencias notificadas dentro de un mismo país deben evaluarse con cuidado. Es muy probable que en un futuro la situación se complique por la llegada de la cepa Vibrio cholerae 0139 a América Latina, situación que genera la necesidad de distinguir entre ella y la cepa 01, que es la predominante.

Para efectos de simplificación y para lograr la amplia aceptación y extensa dioulgación de la información sobre los casos, se recomiendan las siguientes definiciones: caso confirmado de cólera 01: infección por V. cholerae 01 toxígeno, confirmada por métodos de laboratorio, en cualquier persona con diarrea. Caso confirmado de cólera 0139: infección confirmada por V. cholerae 0139 toxígeno, confirmada por un laboratorio, en cualquier persona con diarrea. Caso clínico de cólera: diarrea acuosa de carácter agudo en una persona mayor de 5 años que busca tratamiento. Defunción atribuible al cólera: defunción durante la semana inmediatamente posterior al comienzo de la diarrea en una persona con cólera confirmado o diagnosticado según la definición clínica. Paciente hospitalizado con cólera: persona con cólera confirmado o diagnosticado según la definición clínica que pasa un mínimo de 12 horas en un centro de atención para el tratamiento de la enfermedad.

\footnotetext{
1 Se publicó en inglés en el Bulletin of the Pan American Health Organization, Vol. 30, No. 2, 1996, con el título "Epidemic cholera in Latin America, 19911993: implications of case definitions used for public health surveillance".

2 Centros para el Control y la Prevención de Enfermedades, Atlanta, Georgia, Estados Unidos de
}

América. Dirección postal: Systems Operations and Information Branch, Division of Surveillance and Epidemiology, Epidemiology Program Office, MS C-08, Centers for Disease Control and Prevention, Atlanta, GA 30333, EUA. Las solicitudes de separatas y toda la correspondencia referente a este artículo deben enviarse a Robert Tauxe a la dirección anterior.
3 Organización Panamericana de la Salud, División de Prevención y Control de Enfermedades, Washington, DC, EUA.

4 Organización Panamericana de la Salud, División de Salud y Desarrollo Humano, Washington, DC, EUA.

5 Organización Panamericana de la Salud, Director Adjunto, Washington, DC, EUA. 
La pandemia de cólera causada por Vibrio cholerae 01 toxígeno, biotipo El Tor, llegó a América Latina en enero de 1991 (1, 2). A excepción del Uruguay, todos los países de Centromérica y de América del Sur ya estaban afectados a fines de 1993, y desde entonces el cólera ha seguido constituyendo un problema de salud pública importante en América Latina. La necesidad de observar muy detenidamente el desenvolvimiento de esta epidemia activa resalta la importancia de emplear un sistema de vigilancia oportuno y efectivo.

Después de la llegada del cólera al Perú, la OPS pidió a los epidemiólogos latinoamericanos que presentaran informes semanales del número de casos de cólera, del número de casos hospitalizados y del número de defunciones por cólera en sus respectivas jurisdicciones. Este procedimiento permitió que la OPS y otras partes interesadas vigilaran la epidemia a medida que se extendía por la Región.

En abril y mayo de 1991 se celebraron reuniones con epidemiólogos de todos los países latinoamericanos para tratar de llegar a una definición de casos estandarizada de la enfermedad. Pese a haberse uniformado la definición, varios países siguieron usando las definiciones que siempre habían empleado. Por consiguiente, este elemento fundamental de la vigilancia (la definición de lo que constituye un caso) siguió formulándose individualmente en cada país.

En este resumen se presentan las diversas definiciones de casos de cólera empleadas por los países de América Latina que se han visto afectados por la epidemia. También se da el número de casos de cólera y de defunciones atribuibles a la enfermedad (según datos notificados a la OPS en 1993 por los países latinoamericanos) y se describen algunas tendencias regionales que ha mostrado la incidencia del cólera.

\section{MATERIALES Y MÉTODOS}

De 1991 a 1993, el Ministerio de Salud de cada país latinoamericano recogió información sobre el número de casos de cólera y de hospitalizaciones y defunciones atribuibles a la enfermedad. Estos datos fueron transmitidos al representante de la OPS/OMS, que a su vez los envió a la sede de la OPS en Washington, DC, Estados Unidos de América. Algunos países proporcionaron datos solo por territorio geográfico; otros por territorio y por localidad. Casi todos notificaron a la OPS los totales semanales por correo postal, facsímil o correo electrónico. Unos cuantos países notificaron solo los totales acumulativos y otros con tasas de incidencia de cólera muy bajas, como el Paraguay, enviaron datos solo cuando se detectaron casos de cólera.

En octubre de 1993 enviamos un cuestionario sobre las definiciones de casos de cólera a los 20 países de Centroamérica y de América del Sur que habían notificado la presencia de $V$. cholerae 01 a la OPS desde 1991 y también al Uruguay (único país suramericano que no había notificado ningún caso). En el cuestionario se pedía la definición de un caso de cólera notificado (ya fuera confirmado, probable, sospechado o llamado de alguna otra forma) y de las hospitalizaciones y defunciones atribuibles a la enfermedad. Se envió el cuestionario al Representante de la OPS/OMS en cada país, que lo remitió al Ministerio de Salud. Después de haber enviado los cuestionarios, se hicieron llamadas telefónicas a todos los Representantes que no habían remitido sus respuestas a fines de noviembre de 1993. En el caso de los países centroamericanos cuyas respuestas no se recibieron se emplearon las pautas para la vigilancia del cólera que se publicaron en el informe centroamericano de 1993 (3).

\section{RESULTADOS}

\section{Casos notificados}

Como indica el cuadro 1, los países de América Latina notificaron casi un millón (948 429) de casos de cólera entre 1991 y 1993. Murieron 7955 de los afectados, con lo que se obtiene una tasa de letalidad general de 0,8\%. En 1993 se notificaron 204524 casos; de ellos, 1558 fallecieron, lo cual también corresponde a una tasa de letalidad de 0,8\%. A excepción del Uruguay, todos los países notificaron casos de cólera en el trienio examinado. Desde los comienzos de la epidemia, el Perú ha notificado el mayor número de casos de cólera al año y, hasta 1993, la incidencia más alta de la enfermedad. Sin embargo, en 1993 Guatemala notificó la mayor incidencia y ocupó el tercer lugar entre los países con el mayor número de casos en la Región (figura 1). El Brasil, como la mayor parte de los países centroamericanos, también notificó un aumento anual progresivo del número de casos. Las curvas epidémicas de Guatemala y Nicaragua (figura 2) ilustran estas últimas tendencias.

Durante el mismo período se notificaron tendencias descendentes en varios países suramericanos, como ilustran los ejemplos de Perú y Bolivia, que son bastante típicos (figura 2). La tendencia descendente más marcada se registró en Colombia, donde se notificaron 15129 casos en 1992 y solo 230 en 1993. Presuntamente, la exactitud de las notificaciones que dieron origen a estos datos de Colombia ha sido verificada (Víctor Cárdenas, comunicación personal, septiembre de 1994), aunque no se publicaron datos oficiales que confirmaran la tendencia.

\section{Definiciones de casos}

Las respuestas a los cuestionarios y las definiciones publicadas proporcionaron las definiciones de casos de cólera empleadas por 17 países latinoamericanos. Los cuadros 2 a 4 muestran las diversas definiciones que se aplicaban en 1993 para designar casos de cólera en general, casos de cólera en pacientes hospitalizados y defunciones por cólera. En seis de los 17 países (cuadro 2) la presencia de V. cholerae 01 confirmada por el laboratorio (aun en ausencia de síntomas) fue suficiente para que la enfermedad de la persona infectada se clasificara como un caso de cólera, mientras que en otros nueve países la confirmación más la presencia de síntomas eran requisitos para esta designación. En tres países (Belice, 
CUADRO 1. Número total de casos de cólera y de defunciones por la enfermedad notificados anualmente a la Organización Panamericana de la Salud, por país, de 1991 a 1993. Entre los datos de 1993 también se incluyen las tasas de letalidad (TL) y las cifras de incidencia (casos por 100000 habitantes), según estimaciones de población derivadas del Statistical Yearbook de las Naciones Unidas para 1993 (4)

\begin{tabular}{|c|c|c|c|c|c|c|c|c|}
\hline \multirow[b]{2}{*}{ País } & \multicolumn{2}{|c|}{1991} & \multicolumn{2}{|c|}{1992} & \multicolumn{2}{|c|}{1993} & \multirow[b]{2}{*}{ Incidencia } & \multirow[b]{2}{*}{$\mathrm{TL}(\%)$} \\
\hline & Casos & Defunciones & Casos & Defunciones & Casos & Defunciones & & \\
\hline \multicolumn{9}{|l|}{ América del Sur: } \\
\hline Bolivia & 206 & 12 & 22260 & 383 & 10134 & 254 & 126,0 & 2,5 \\
\hline Brasil & 2101 & 26 & 30054 & 359 & 56286 & 607 & 35,0 & 1,1 \\
\hline Chile & 41 & 2 & 73 & 1 & 32 & 0 & 0,2 & 0,0 \\
\hline Guyana & 0 & 0 & 556 & 8 & 66 & 2 & 8,2 & 3,0 \\
\hline Paraguay & 0 & 0 & 8 & 0 & 3 & 0 & $<0,1$ & 0,0 \\
\hline Perú & 322562 & 2909 & 210836 & 727 & 71448 & 575 & 295,0 & 0,8 \\
\hline Suriname & 0 & 0 & 12 & 1 & 0 & 0 & 0,0 & - \\
\hline Uruguay & 0 & 0 & 0 & 0 & 0 & 0 & 0,0 & - \\
\hline Venezuela & 13 & 2 & 2842 & 68 & 409 & 10 & 1,9 & 2,4 \\
\hline Guatemala & 3652 & 50 & 15686 & 207 & 30604 & 306 & 305,0 & 1,0 \\
\hline Honduras & 17 & 0 & 388 & 17 & 2290 & 64 & 40,0 & 2,8 \\
\hline México & 2690 & 34 & 8162 & 99 & 10712 & 193 & 12,0 & 1,8 \\
\hline Nicaragua & 1 & 0 & 3067 & 46 & 6631 & 220 & 155,0 & 3,3 \\
\hline Panamá & 1178 & 29 & 2416 & 49 & 42 & 4 & 1,6 & 9,5 \\
\hline Total & 391708 & 4002 & 352197 & 2395 & 204524 & 1558 & 1204,1 & 0,8 \\
\hline
\end{tabular}

FIGURA 1. Incidencia de casos de cólera notificada en 1993 en América del Sur, Centroamérica y México, por país

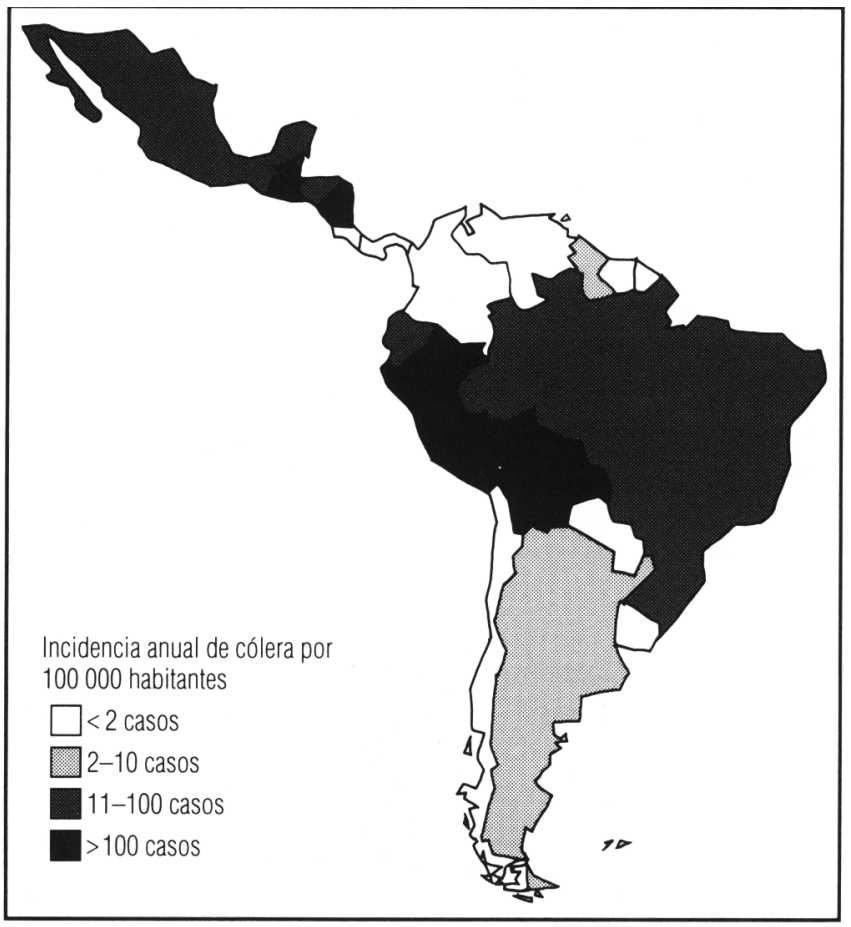

Costa Rica y México) se exigía que todos los casos se confirmaran mediante estudios de laboratorio. Por otra parte, un país (El Salvador) definió el cólera sobre la base exclusiva de criterios clínicos. En ocho países cuyas definiciones estaban basadas en criterios clínicos (para casos sin confirmación por un laboratorio), ${ }^{6}$ un individuo con cólera tenía que satisfacer uno de dos criterios epidemiológicos: a) haber entrado en contacto con una persona con cólera confirmado por un laboratorio o b) haber sufrido exposición en una zona donde se hubiera confirmado en el laboratorio la presencia de $V$. cholerae 01.

Las definiciones empleadas por diferentes países para designar casos de cólera en pacientes hospitalizados también fueron distintas (cuadro 3). En siete países de un total de 11, la pre-

Bolivia, Brasil, Chile, Colombia, Honduras, Nicaragua, Paraguay y Uruguay. 
FIGURA 2. Número de casos de cólera notificados por quincena en Bolivia, Guatemala, Nicaragua y Perú, 1991 a 1993
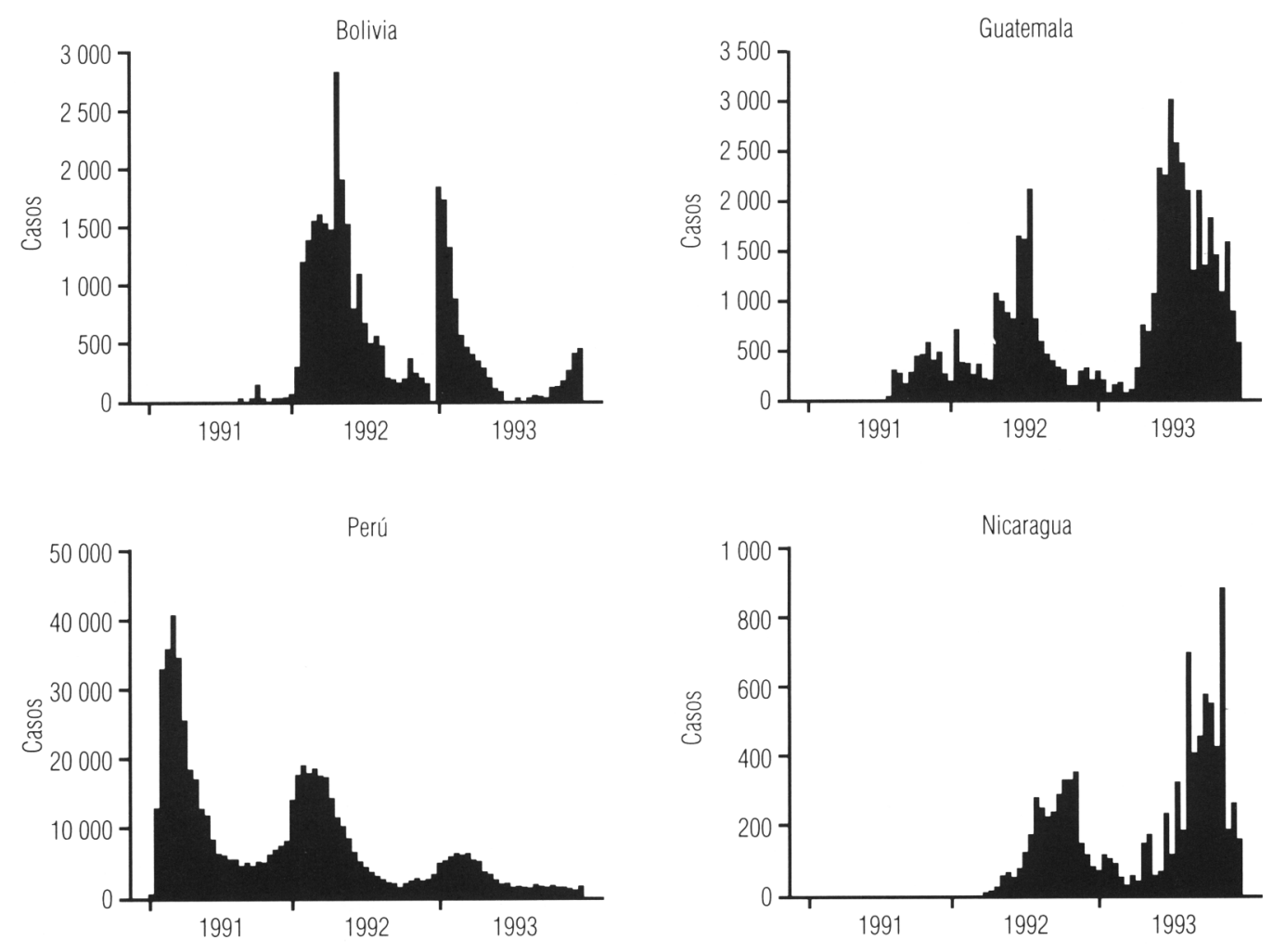

sencia o ausencia de hospitalización dependió del tiempo que el paciente hubiera pasado en un centro de tratamiento u hospital. Otros cuatro países no tenían ningún requisito temporal, de modo que si una persona con cólera entraba a un centro de atención de salud en busca de tratamiento, ello de por sí bastaba para que se le considerara hospitalizada por cólera.

Para definir las defunciones por cólera (cuadro 4) se aplicaron criterios similares a los empleados para definir los casos de la enfermedad. Ningún país especificó si el número de días transcurridos desde el inicio de la enfermedad se había usado o no para ayudar a determinar si la muerte en cuestión había sido producida por el cólera.

\section{DISCUSIÓN}

El sistema de vigilancia de la OPS/OMS ha servido para determinar las tendencias de la incidencia de enfermedad en ciertos países. A fines de 1993, el número de casos de cólera notificados mostraba una tendencia descendente en la mayor parte de los países suramericanos, aunque ascendía en muchos países de Centroamérica. Esta información ha resultado útil para alertar a los viajeros o aplacar sus temores y para seguir de cerca el rápido avance o declive de la epidemia.

Sin embargo, varían mucho las definiciones empleadas por diferentes países para notificar casos de cólera. Es muy probable que los países con cólera epidémico que notifican solo casos confirmados por un laboratorio tengan una gran subnotificación, puesto que se les escapa la enfermedad en personas cuyas heces no se someten a cultivo y cuyo caso, por ende, se queda sin notificar. Esos países tampoco pueden contar entre las defunciones por cólera las de personas que han fallecido de la enfermedad sin haberse sometido a un cultivo, cir- cunstancia que podría disminuir artificialmente la tasa de letalidad. Por añadidura, los agentes de salud pública en otros países que aplican a casos de cólera definiciones clínico-epidemiológicas complejas pueden tener dificultad en emplear dichas definiciones. Por lo tanto, es posible que también subnotifiquen el número de casos.

Cabe señalar que aunque este informe presenta solo las definiciones de casos de cólera empleadas a partir de octubre de 1993, algunas han cambiado. Por ejemplo, desde fines de 1993 Guatemala ha usado las definiciones recomendadas a continuación (5). En términos generales, sin embargo, las variaciones que han sufrido estas definiciones ponen de manifiesto la dificultad de hacer comparaciones generales entre países (o incluso de calcular la carga de morbilidad y de evaluar la calidad de la atención tomando como base las tasas de letalidad) y demuestran que aun las tendencias notificadas dentro de un 
CUADRO 2. Criterios aplicados a partir de octubre de 1993 en 21 países latinoamericanos para designar casos de enfermedad que se ciñen a la definición de cólera usada para fines de la vigilancia. SX = Es preciso que los casos confirmados en el laboratorio tengan síntomas, además de resultados de laboratorio positivos

\begin{tabular}{|c|c|c|c|c|}
\hline \multirow[b]{2}{*}{ País } & \multirow[b]{2}{*}{$\begin{array}{l}\text { Confirmados } \\
\text { en el laboratorio }\end{array}$} & \multicolumn{3}{|c|}{ Definición clínica } \\
\hline & & $\begin{array}{l}\text { Con criterios } \\
\text { epidemiológicos }\end{array}$ & $\begin{array}{c}\text { Con criterios clínicos } \\
\text { únicamente }\end{array}$ & $\begin{array}{c}\text { Con criterios } \\
\text { específicos por edad }\end{array}$ \\
\hline \multicolumn{5}{|c|}{ América del Sur: } \\
\hline Bolivia & SX & $x$ & & Síc \\
\hline Brasil & SX & $X$ & & $\operatorname{Sí}^{d}$ \\
\hline Ecuador & $x$ & $x$ & $x$ & $\mathrm{No}^{\mathrm{e}}$ \\
\hline \multicolumn{5}{|c|}{ Guayana Francesa ${ }^{b}$} \\
\hline Guyana & $X$ & & $x$ & Síc \\
\hline Paraguay & SX & $x$ & & No \\
\hline Perú & $x$ & & $x$ & No \\
\hline \multicolumn{5}{|l|}{ Suriname $^{b}$} \\
\hline \multirow{2}{*}{\multicolumn{5}{|c|}{ Venezuela ${ }^{b}$}} \\
\hline & & & & \\
\hline El Salvador & & & $x$ & No \\
\hline Guatemala & sX & $x$ & $X$ & No \\
\hline Honduras & SX & $x$ & & No \\
\hline México & SX & & & No \\
\hline Nicaragua & SX & $X$ & & No \\
\hline Panamá & $x$ & $x$ & $x$ & No \\
\hline
\end{tabular}

mismo país deben evaluarse cuidadosamente. Por otro lado, la mayor parte de las definiciones empleadas en los países han permanecido relativamente estables. (La comparación directa de las tasas suele tener más validez en países que usan definiciones similares para designar los casos.)

La pandemia causada por $V$. cholerae 0139 que se ha propagado por toda el Asia (6-8) todavía (es decir, a comienzos de 1996) no ha afectado a América Latina. Sin embargo, un caso de la infección fue importado a Estados Unidos en 1993 (9), otros 17 llegaron en 1994 (10), y es probable que $V$. cholerae 0139 también aparezca en América Latina. De ser así, habrá que montar una vigilancia en los laboratorios de una muestra de lugares centinela para distinguir entre el cólera causado por la cepa 01 y el producido por la cepa 0139. Los informes generados por esta vigilancia serían más útiles si dentro del total de casos confirmados se incluyeran listas separadas para los casos de infección confirmada por la cepa 0139. Aunque esta última infección posee características clínicas $\mathrm{y}$ epidemiológicas similares a las de la infección por la cepa 01 , la notificación del serotipo específico tendrá importancia crítica para poder seguir la trayectoria de esta nueva ola pandémica, alertar a los países vecinos de su llegada e idear medidas preventivas específicas. Los Centros para el Control y la Prevención de Enfermedades en los Estados Unidos han publicado un manual de métodos de laboratorio estandarizados para el diagnóstico de cólera y entre ellos figuran los procedimientos utilizados para identificar la cepa 0139 (11).

La práctica oportuna y acertada de la vigilancia del cólera en los ámbitos nacional e internacional refuerza las medidas de control de la enfermedad y genera la información necesaria para proporcionar ayuda internacional inmediata a los países más afectados. No obstante, para que la vigilancia del cólera sea eficaz, la notificación de casos debe ser sencilla y gozar de aceptación general y sus resultados deben difundirse ampliamente y de manera oportuna (12-14). Las definiciones de los casos que requieren notificación deben, asimismo, ser sencillas, tener una amplia aceptación y divulgarse 
CUADRO 3. Criterios empleados a partir de octubre de 1993 en 21 países latinoamericanos en lo referente al tiempo que debe permanecer ingresada una persona en un hospital u otro centro de atención para ser clasificada como paciente de cólera hospitalizado

\begin{tabular}{|c|c|c|c|c|}
\hline \multirow[b]{2}{*}{ País } & \multicolumn{4}{|c|}{ Duración de la estancia en el centro de atención u hospital } \\
\hline & No hay ningún requisito & $>12$ horas & $>24$ horas & Se desconoce ${ }^{a}$ \\
\hline \multicolumn{5}{|l|}{ América del Sur: } \\
\hline Argentina & & & & $X$ \\
\hline Bolivia & & $X$ & & \\
\hline Brasil $^{b}$ & & $x$ & & \\
\hline Chile & & & & $X$ \\
\hline Colombiac $^{c}$ & & $X$ & $x$ & \\
\hline Ecuador & & $x$ & & \\
\hline Guayana Francesa & & & & $X$ \\
\hline Guyana & $X$ & & & \\
\hline Paraguayd & & & & \\
\hline Perú & & & $X$ & \\
\hline Suriname & & & & $X$ \\
\hline Uruguay d & & & & \\
\hline Venezuela & & & & $X$ \\
\hline \multicolumn{5}{|c|}{ México y Centroamérica: } \\
\hline Belice & $X$ & & & \\
\hline Costa Rica & & & & $X$ \\
\hline El Salvador & & & $X$ & \\
\hline Guatemala & & & & $X$ \\
\hline Honduras & $X$ & & & \\
\hline México & & & $X$ & \\
\hline Nicaragua & & & & $X$ \\
\hline Panamá & $X$ & & & \\
\hline
\end{tabular}

extensamente $(12,13)$. En países latinoamericanos recomendamos el empleo de las siguientes definiciones para la notificación de casos de cólera:

- Caso confirmado de cólera 01: infección por $V$. cholerae 01 toxígeno, confirmada por un laboratorio, en cualquier persona con diarrea.

- Caso confirmado de cólera 0139: infección por V. cholerae 0139 toxígeno, confirmada por un laboratorio, en cualquier persona con diarrea.

- Caso clínico de cólera: diarrea acuosa aguda en una persona de 5 años de edad o más que busca tratamiento.

- Defunción atribuible al cólera: defunción de una persona con cólera confirmado o diagnosticado según la definición clínica, en el transcurso de la semana inmediatamente posterior al comienzo de la diarrea.
- Paciente hospitalizado con cólera: persona con cólera confirmado o diagnosticado según la definición clínica, que pasa un mínimo de 12 horas en un centro de atención de salud para el tratamiento de la enfermedad.

Cabe señalar que los casos diagnosticados según parámetros clínicos no se han de notificar junto con los que han sido confirmados y que el término confirmado se usa solo para casos con confirmación por un laboratorio. En el contexto de la epidemia peruana, la definición clínica de la enfermedad (que es sencilla y fácil de usar) ha mostrado tener una especificidad para el cólera de 80 a 90\% (15-17). Sin embargo, dada la variedad de causas de diarrea en niños menores de 5 años, la definición clínica de un caso carece de especificidad para el cólera en este grupo de edad y, por ende, solo debe aplicarse a personas mayores.

Es posible, por añadidura, que la definición clínica sea poco útil en países donde el cólera es una causa de diarrea poco frecuente. Como ello sugiere, la confirmación de todos los casos de cólera o de algunos por un laboratorio es importante en ciertos países y también tendrá importancia si $V$. cholerae 0139 llega a penetrar en América Latina.

Recomendamos el uso de una definición estandarizada para pacientes hospitalizados con cólera en países donde se lleva la cuenta de los ingresos. Aun así, la variedad de las prácticas clínicas en diferentes países nos hace pensar que la notificación internacional del número de pacientes hospitalizados no tendrá la uniformidad suficiente para poder hacer comparaciones. 
CUADRO 4. Criterios aplicados a partir de octubre de 1993 por 21 países latinoamericanos para definir las defunciones atribuibles al cólera, en el contexto de las actividades de vigilancia

\begin{tabular}{|c|c|c|c|c|}
\hline \multirow[b]{2}{*}{ País } & \multirow[b]{2}{*}{$\begin{array}{l}\text { Confirmados } \\
\text { en el laboratorio }\end{array}$} & \multicolumn{3}{|c|}{ Definición de casos clínicos } \\
\hline & & $\begin{array}{l}\text { Con criterios } \\
\text { epidemiológicos }\end{array}$ & $\begin{array}{c}\text { Con criterios clínicos } \\
\text { únicamente }\end{array}$ & $\begin{array}{c}\text { Tasa de letalidad } \\
\text { en } 1993\end{array}$ \\
\hline \multicolumn{5}{|l|}{ América del Sur: } \\
\hline Argentina $^{b}$ & & & & 1,6 \\
\hline Bolivia & & $\mathrm{X}$ & & 2,5 \\
\hline Brasil & & $X$ & & 1,1 \\
\hline Chile & $\mathrm{X}$ & $x$ & & 0,0 \\
\hline Colombia & & $X$ & & 1,7 \\
\hline Ecuador & $x$ & $X$ & & 1,1 \\
\hline Guayana Francesa ${ }^{b}$ & & & & 0,0 \\
\hline Guyana & & & $\mathrm{X}$ & 3,0 \\
\hline Paraguay & $\mathrm{X}$ & & & 0,0 \\
\hline Perú & & & $\mathrm{X}$ & 0,8 \\
\hline Suriname ${ }^{b}$ & & & & - \\
\hline Uruguayc $^{c}$ & & & & - \\
\hline Venezuela $^{b}$ & & & & 2,4 \\
\hline \multicolumn{5}{|l|}{ México y Centroamérica: } \\
\hline Belice & $\mathrm{X}$ & $\mathrm{X}$ & & 2,2 \\
\hline Costa Ricab & & & & 0,0 \\
\hline El Salvador & & & $X$ & 0,2 \\
\hline Guatemalab $^{b}$ & & & & 1,0 \\
\hline Honduras & $\mathrm{X}$ & $\mathrm{X}$ & & 2,8 \\
\hline México & $\mathrm{X}$ & & & 1,8 \\
\hline Nicaragua $^{\mathrm{b}}$ & & & & 3,3 \\
\hline Panamá & & & $\mathrm{X}$ & 9,5 \\
\hline
\end{tabular}

\section{REFERENCIAS}

1. Pan American Health Organization. Cholera situation in the Americas: an update. Epidemiol Bull 1991;12:11.

2. Tauxe R, Seminario L, Tapia R, Libel M. The Latin American cholera epidemic. En: Wachsmuth IK, Blake PA, Olsvik O, eds. Vibrio cholerae and cholera: molecular to global perspectives. Washington, DC: American Society for Microbiology; 1994:321-344.

3. Salcedo J. El cólera en Centroamérica. Guatemala: Organización Panamericana de la Salud; 1993. (Documento mimeografiado).

4. United Nations. Statistical yearbook. [38a impresión]. New York: UN; 1993.

5. Guatemala, Ministerio de Salud Pública y Asistencia Social, Dirección General de Servicios de Salud, División de Vigilancia y Control de Enfermedades, Departamento de Vigilancia Epidemiológica. Normas y procedimientos para la vigilancia del cólera. Guatemala: MSPAS; 1994.

6. World Health Organization. Epidemic diarrhea due to Vibrio cholerae non-01. Wkly Epidemiol Rec 1993;68:141-142.

7. Ramamurthy T, Garg S, Sharma R, et al. Emergence of novel strain of Vibrio cholerae with epidemic potential in southern and eastern India. [Carta]. Lancet 1993;341:703-704.
8. Albert MJ, Siddiguqi AK, Islam MS, et al. Large outbreak of clinical cholera associated with a newly described toxigenic Vibrio cholerae non01 in Bangladesh. [Carta]. Lancet 1993;341:704.

9. Centers for Disease Control and Prevention. Imported cholera associated with a newly described toxigenic Vibrio cholerae O139 strain: California, 1993. Morbid Mortal Wkly Rep 1993;42:501-503.

10. Boyce TG, Mintz EM, Greene KD, et al. Vibrio cholerae $\mathrm{O} 139$ Bengal infections among tourists to Southeast Asia: an intercontinental foodborne outbreak. J Infect Dis 1995;172:1401-1404.

11. United States, Department of Health and Human Services, Public Health Service, Centers of Disease Control and Prevention. Laboratory methods for the diagnosis of Vibrio cholerae. Atlanta: CDC; 1994

12. Centers for Disease Control and Prevention. Surveillance for cholera: Cochabamba Department, Bolivia, January-June 1992. Morbid Mortal Wkly Rep 1993;42:636-639.

13. Vugia DJ, Koehler JE, Ries AA. Surveillance for epidemic cholera in the Americas: an assessment. Morbid Mortal Wkly Rep 1992; 41:27-34.

14. Vugia DJ. Cholera surveillance. En: Wachsmuth IK, Blake PA, Olsvik O, eds. Vibrio cholerae and cholera: molecular to global perspectives. Washington, DC: American Society for Microbiology; 1994:371-378.

15. Serdlow DL, Mintz ED, Rodríguez M, et al. Waterborne transmission of epidemic cholera in Piura, Peru: lessons for a continent at risk. J Infect Dis 1992;340:28-33.

16. Ries AA, Vugia DJ, Beingolea L, et al. Cholera in Piura, Peru: a modern urban epidemic. J Infect Dis 1992;166:1429-1433.

17. Vugia DJ, Rodríguez M, Vargas R, et al. Epidemic cholera in Trujillo, Peru, 1992: utility of a clinical case definition and shift in Vibrio cholerae 01 serotype. Am J Trop Med Hyg 1994; 50:566-569.

Manuscrito recibido el 4 de abril de 1995. Aceptado para publicación en el Boletín de la Oficina Sanitaria Panamericana y en el Bulletin of the Pan American Health Organization el 5 de febrero de 1996. 
ABSTRACT This report presents the various cholera case definitions used by the affected countries of Latin America, shows the numbers of cholera cases and deaths attributable to cholera (as reported by Latin American countries to PAHO through 1993), and Epidemic cholera in Latin America, 1991-1993: implications of case definitions used for public health surveillance describes some regional trends in cholera incidence. The information about how cholera cases were defined was obtained from an October 1993 PAHO questionnaire.

In all, 948429 cholera cases were reported to PAHO by affected Latin American countries from January 1991 through December 1993, the highest annual incidences being registered in Peru (1991 and 1992) and Guatemala (1993). The case-fatality rate over the three-year period, and also in 1993, was 0.8\%. A general downward trend in the incidence of cholera was observed in most South American countries, while the incidence increased in most Central American countries.

A good deal of variation was noted in the definitions used for reporting cholera cases, hospitalized cholera cases, and cholera-attributable deaths. Because of these variations, broad intercountry comparisons (including disease burden calculations and care quality assessments based on case-fatality rates) are difficult to make, and even reported trends within a single country need to be evaluated with care. The situation is likely to be complicated in the future by the arrival of $V$. cholerae 0139 in Latin America, creating a need to distinguish between it and the prevailing 01 strain.

For purposes of simplicity, wide acceptance, and broad dissemination of case data, the following definitions are recommended: Confirmed case of 01 cholera: laboratory-confirmed infection with toxigenic $V$. cholerae 01 in any person who has diarrhea. Confirmed case of 0139 cholera: laboratory-confirmed infection with toxigenic $V$. cholerae 0139 in any person who has diarrhea. Clinical case of cholera: acute watery diarrhea in a person over 5 years old who is seeking treatment. Death attributable to cholera: death within one week of the onset of diarrhea in a person with confirmed or clinically defined cholera. Hospitalized patient with cholera: a person who has confirmed or clinically defined cholera and who remains at least 12 hours in a health care facility for treatment of the disease.

\title{
Cursos de posgrado en epidemiología clínica
}

Fechas: $\quad$ junio, julio y septiembre de 1997

Lugar: $\quad$ Ciudad de La Habana, Cuba

El Hospital "Hermanos Ameijeiras" será la sede de tres cursos de posgrado en epidemiología clínica para médicos, enfermeras y otros profesionales de la salud. En el primero, que se celebrará del 30 de junio al 4 de julio, se examinarán el papel de la investigación en la medicina clínica contemporánea y los procedimientos básicos para planificar una investigación. El segundo, que cubrirá los elementos fundamentales de la epidemiología clínica, tendrá lugar del 7 al 18 de julio y se centrará en la evaluación de pruebas y estrategias diagnósticas, en la evaluación de medidas terapéuticas y preventivas y en los factores que influyen en el pronóstico de las enfermedades. El tercer curso se ofrecerá del 22 al 26 de septiembre y estará dedicado al diseño de un ensayo clínico y al análisis estadístico de sus resultados.

\author{
Información: \\ Dra. Rosa Jiménez Paneque \\ Hospital "Hermanos Ameijeiras" \\ Sección de Investigaciones \\ San Lázaro 701 \\ Ciudad Habana 3, 10300, Cuba \\ Teléfono: (53) (7) 616344 \\ Fax: (53) (7) 335036 \\ Correo electrónico: \\ fa@hha.sld.cu \\ Icsilva@infomed.sld.cu
}

